\title{
Rede Docente Assistencial UFSC/SMS de Florianópolis: Reflexos da Implantação dos Projetos Pró-Saúde I e II
}

\author{
The Academic Healthcare System under the \\ Federal University in Santa Catarina and the \\ Florianópolis Municipal Health Secretariat: \\ Effects of the Pro-Health I and II Projects
}

\author{
Kenya Schmidt Reibnitz \\ Maria Francisca dos Santos Daussy II \\ Carlos Alberto Justo da Silva ${ }^{I}$ \\ Marynes Terezinha Reibnitz ${ }^{\text {II }}$ \\ Daiana Kloh
}

\author{
PALAVRAS-CHAVE: \\ - Política de Saúde; \\ - Integração Docente- \\ Assistencial; \\ - Ensino Superior; \\ - Sistema Único de Saúde.
}

\author{
KEYWORDS: \\ - Health Policy; \\ - Teaching Care Integration \\ Services; \\ - Education Higher; \\ - Single Health System.
}

\section{Recebido em: 01/10/2010}

Aprovado em: 19/11/2010

REVISTA BRASILEIRA DE EDUCAÇÃO MÉDICA 68 36(1, Supl. 2): $687.75 ; 2012$
${ }^{I}$ Universidade Federal de Santa Catarina, Florianópolis, SC, Brasil.

"Secretaria Municipal de Saúde de Florianópolis, Florianópolis, SC, Brasil; Universidade Federal de Santa Catarina, Florianópolis, SC, Brasil III Secretaria Municipal de Saúde de Florianópolis, Florianópolis, SC, Brasil 


\section{INTRODUÇÃO}

A Universidade Federal de Santa Catarina (UFSC) tem desenvolvido experiências institucionalizadas de articulação com o Sistema Único de Saúde (SUS), nos níveis de graduação e pós-graduação (Residência em Medicina de Família e Comunidade e Residência Multiprofissional em Saúde da Família) e educação permanente. Essas atividades passaram a se concretizar mais intensivamente com a Rede Docente Assistencial (RDA), uma parceria da UFSC/Centro de Ciências da Saúde (CCS) e o Hospital Universitário Polydoro Hernani de São Thiago (HU) com a Secretaria Municipal da Saúde de Florianópolis (SMS de Florianópolis), que, além dos cursos próprios CCS, passou a dialogar também com os demais cursos da saúde que compõem a equipe multidisciplinar. Tal prática tem evidenciado não apenas os enormes desafios a serem enfrentados, mas também a produtividade desse tipo de articulação para a produção de conhecimento e a formação de profissionais engajados no amplo processo de mudança do setor da saúde.

Um dos maiores desafios está em lidar com a dialética entre a prática educacional e o cuidado em saúde. Ela precisa ser entendida como promotora de ações inovadoras, pois a educação em serviço evidencia o potencial pedagógico do trabalho, proporcionando uma aprendizagem contínua e de natureza participativa, articulando o fazer, o educar, o saber e o conviver.

Nessa perspectiva, faz-se necessária a aproximação entre as instituições formadoras e o mundo do trabalho, fortalecendo a integração ensino-serviço, por meio da compreensão acerca do processo de trabalho e da realidade social, promovendo no ensino uma inovação pedagógica. $\mathrm{O}$ trabalho passa a ser a condição natural da aprendizagem, provocando nos atores do processo uma ruptura com a acomodação e a alienação, possibilitando experiências contextualizadas com o mundo do trabalho e estimulando o exercício da interdisciplinaridade. As práticas profissionais, tanto dos docentes como dos profissionais do serviço, passam a ser compreendidas como um processo solidário de ação-reflexão-ação, de indagação e experimentação, no qual todos ensinam e também aprendem, intervindo para facilitar a aprendizagem e não para impor nem substituir a compreensão.

É importante destacar que entre os princípios do SUS coloca-se o da integralidade, que busca articular os dois modelos: as ações curativas e as preventivas (individuais e coletivas), respondendo universalmente e resolutivamente às necessidades sociais em saúde. Além disso, "absorve" o entendimento de fazer saúde como ação coletiva e não como um trabalho fragmentado, em que o conhecimento é instrumento de poder e de adaptação social, de defesa ao já instituído.
A partir de uma prática problematizadora da realidade local e envolvendo os diversos segmentos humanos e estruturais no processo, é possível articular o sistema de saúde e as instituições de ensino, reorganizando, assim, os serviços e os processos formativos, transformando as práticas educativas e assistenciais.

Nesse sentido, deve-se pensar na educação não como mera reprodução do conhecimento, mas como um processo de emancipação dos sujeitos. E, para o alcance dessa emancipação, é necessário definir que tipo de educação se pretende para a área da saúde. Uma educação reduzida à transmissão dos conhecimentos prontos, limitada à formação profissional técnica, ou uma educação em que valores como participação, ética, solidariedade e esperança sejam também fundamentais.

Segundo Asmann e Sung², a educação se transformou na tarefa emancipatória mais significativa e, para tal, articula teoria e prática, ensino e serviço, a competência profissional à competência social, em que a sensibilidade social e a sensibilidade ética estão presentes para construção de uma outra práxis pedagógica, a práxis criadora.

Na práxis criadora, o trabalhador alia seu lado objetivo ao subjetivo; usa seu poder de criação enquanto atividade racional, pois o trabalho traz consigo um projeto preconcebido, o que revela o grau de consciência do sujeito. A práxis criadora tem um caráter inovador, traz à tona uma nova realidade prática. Esse poder de criação ocorre em função de uma necessidade - a finalidade do trabalho ${ }^{3}$.

Dessa maneira, passa-se a entender que o trabalho traz em seu cerne o poder da criação, não sendo apenas um ato de repetição. Como atividade, ele é consciente e racional, sendo uma atividade que só o homem tem a capacidade de idealizar, projetar o que irá fazer, a partir de um projeto preconcebido do produto final do seu "próprio trabalho". Somente o ser humano tem essa potencialidade transformadora, que permite "recuperar, historicamente, a concepção do trabalho como um componente que, ultrapassando a mera atividade instintiva dos animais, converteu-se na força que criou a espécie humana e uma força pela qual a humanidade criou o mundo que conhecemos"4 (p. 33).

Assim, o espaço pedagógico vai além da sala de aula, ele se contextualiza nos espaços do mundo do trabalho, e, para que isso aconteça, ou seja, para que esse mundo se constitua experiências de aprendizagem, esses espaços precisam ser construídos pelo processo crítico-criativo dos docentes, profissionais e estudantes, com responsabilidades compartilhadas. As situações de aprendizagem são a própria realidade com que se defrontam os estudantes no cotidiano dos serviços. O desafio colocado aos protagonistas nesse processo é o de implementar formas dinâmicas para conectar o mundo da educação com o mundo do trabalho, possibilitando um ensino 
prático-reflexivo e a construção de sujeitos-cidadãos-profissionais capacitados não só para responder, mas também para questionar as diferentes demandas que o mundo do trabalho e suas transformações lhes impõem 5 .

Refletir sobre a prática provoca o estreitamento das relações entre as instituições formadoras e assistenciais, a partir da compreensão acerca do processo de trabalho e da realidade social, e promove a educação permanente.

Segundo Ceccim ${ }^{6}$

A Educação Permanente em Saúde, ao mesmo tempo em que disputa pela atualização cotidiana das práticas, segundo os mais recentes aportes teóricos, metodológicos, científicos e tecnológicos disponíveis, insere-se em uma necessária construção de relações e processos que vão do interior das equipes em atuação conjunta, implicando seus agentes, às práticas organizacionais, implicando a instituição e/ou o setor da saúde, e às práticas interinstitucionais e/ou intersetoriais, implicando as políticas nas quais se inscrevem os atos de saúde. (p.161)

Essa educação permanente permite revelar a complexidade e a articulação das explicações dos diferentes problemas a partir da realidade do processo de trabalho. Torna evidente a necessidade de várias estratégias que, para serem propostas e implementadas, necessitam de articulação com a gestão do sistema. Ou seja, para operar de maneira eficaz, a educação permanente necessita ser tomada como uma estratégia de gestão, propiciando a mobilização de liderança e de recursos para enfrentar os diferentes problemas do cotidiano.

\section{O Contexto Social e Político para Criação da RDA}

O convênio já existente entre a UFSC e a SMS de Florianópolis estabelecia um Programa Docente Assistencial (PDA), fundamentado na lógica multidisciplinar, organizado em uma rede hierarquizada e regionalizada (local) de unidades de saúde de diferentes níveis de complexidade, tendo como finalidade atender à necessidade de formação de profissionais no setor público de saúde, de acordo com os princípios do SUS, procurando articular as necessidades do ensino com as necessidades dos serviços de saúde.

Os objetivos do Sistema-Escola visam ao desenvolvimento de programas de formação e capacitação de profissionais, de acordo com as diretrizes do SUS, em uma área de saúde funcional e geograficamente definida como de integração docente-assistencial, viabilizando-se oficialmente um trabalho com história de mais de 20 anos de experiências extracurriculares ${ }^{7}$.(p.45)
O modelo gerencial era de cogestão entre setores da UFSC (HU, CCS e Departamentos envolvidos fora do CCS) e setores da SMS de Florianópolis (Gabinete do Secretário, Departamento de Ações de Saúde, Regional de Saúde Leste). Os titulares das representações institucionais (diretores e secretário de saúde) constituíam um núcleo gestor de comando político, e os demais representantes dos setores envolvidos compunham um colegiado técnico-administrativo.

Com a ampliação dos serviços e necessidades das instituições parceiras (SMS de Florianópolis e UFSC), algumas fragilidades foram surgindo no PDA, diante do avanço das discussões relacionadas ao processo de trabalho em saúde, a Política de Educação Permanente e a implantação das Diretrizes Curriculares Nacionais para os cursos da área da saúde, dentre as quais se destacam:

- Criação de unidades modelos para a aplicação do processo de ensino-aprendizagem, provocando diferenças no atendimento à população.

- Processos de trabalho diferenciados entre os profissionais da SMS de Florianópolis que atuavam no PDA e os que não atuavam.

- Acúmulo de alunos nos Centros de Saúde (CS) sem uma prévia análise global da capacidade instalada.

- Foco das atividades centradas no ensino e não na assistência.

Perante esse diagnóstico e impulsionados pela atual Política de Formação dos Profissionais da Saúde assumida pelos gestores da SMS de Florianópolis e UFSC, em especial o CCS, passou-se a discutir coletivamente estratégias de fortalecimento dessa parceria. Essa discussão teve por base a perspectiva de ampliação das ações contemplando não só as necessidades do processo de formação, mas também um trabalho mais integrado voltado para a atenção à saúde, esta fundamentada nos princípios do SUS, articulada com as necessidades da formação dos profissionais e o atendimento da população, potencializando a formação no trabalho em saúde, tendo em vista que o conhecimento é construído a partir da realidade.

A ampliação das ações envolveu os demais cursos de graduação da área da saúde, promovendo um trabalho mais articulado com a realidade do serviço e a necessidade de saúde da população. Era premente a necessidade de se pensar estratégias para consolidação da articulação ensino-serviço, ressaltando a importância da comunicação e da negociação na construção de um projeto democrático, suplantando a dicotomia autonomia versus controle e poder, promovendo relações mais fecundas entre trabalhadores, gerentes e usuários.

Nessa perspectiva de ampliação, e fortalecendo a pactuação com as instituições envolvidas, surge a criação da RDA entre a 
UFSC e a SMS de Florianópolis, tendo em vista que um trabalho em rede tem um fundamento básico: o processo de articulação entre pessoas, grupos e instituições com objetivos comuns.

\section{A REDE DOCENTE ASSISTENCIAL - UFSC/SMS DE FLORIANÓPOLIS}

Uma rede impulsiona mudanças estruturais no modo de pensar e agir de um grupo profissional e/ou líderes institucionais e fortalece oportunidades para o surgimento de novas propostas, respeitando o tempo de cada um.

Para implantar uma rede, é necessário tempo para aproximação, para clareamento de necessidades, para definição de objetivos e metas, para instrumentalização de suportes, para institucionalização das ideias e propostas e, finalmente, para a contínua avaliação, redefinições e novos acordos ${ }^{8} \cdot(p .61)$

Em sua fundamentação teórico-filosófica, estabelece os seguintes pressupostos:

- A RDA representa um instrumento facilitador da intermediação entre as estruturas de formação e serviços.

- A ampliação da atenção à saúde está fundamentada nos princípios do SUS, articula-se com as necessidades da formação dos profissionais e o atendimento à população.

- A rede potencializará a formação no trabalho em saúde, tendo em vista que o conhecimento é construído a partir da realidade.

- O conhecimento sistematizado ou desvelado pelos diversos participantes (instituições, profissionais e comunidade) precisa ser colocado à disposição da sociedade em geral e no contexto da saúde em particular.

- A rede promove maior visibilidade do compromisso social da UFSC e da SMS de Florianópolis no contexto da sociedade.

- A rede se efetiva mediante mecanismos de comunicação integrados, dinâmicos e participativos.

\section{OBJETIVOS E METAS}

A criação da RDA entre a UFSC e a SMS de Florianópolis tem como objetivo geral promover a integração ensino, serviço e comunidade, favorecendo a ampliação da atenção à saúde de qualidade e propiciando a formação dos profissionais da saúde voltada para os princípios do SUS.

Objetivos específicos:

- Estabelecer compromisso institucional para encaminhar politicamente a proposta de criação da RDA, oficializando-a e apoiando a sua institucionalização.
- Desenvolver proposta de gestão compartilhada, estabelecendo atribuições e compromissos das instituições envolvidas através de regimento específico.

- Construir coletivamente o processo com os atores envolvidos, respeitadas as especificidades históricas e políticas de cada instituição.

- Criar os espaços necessários para discussão e encaminhamentos de questões e conflitos inerentes ao processo de consolidação da RDA.

- Promover um processo de ensino contextualizado com a realidade da prática.

- Fomentar o desenvolvimento de pesquisas integradas, considerando o contexto sociocultural, compatibilizando o modelo pedagógico-assistencial e as necessidades de saúde da população.

- Estabelecer, de forma compartilhada, um programa para educação permanente dos profissionais de saúde.

- Desenvolver mecanismos de referência e contrarreferência entre as instituições.

Como estratégias para atingir os objetivos propostos, destacam-se o desenvolvimento de ações que permitam:

- atenção ao indivíduo e ao meio ambiente, tanto físico quanto social;

- cuidados de saúde que englobam a promoção, prevenção e atividade curativa, abrangendo todo o ciclo vital;

- articulação com os demais níveis do sistema de saúde por meio de mecanismos efetivos de referência e contrarreferência;

- enfoque na Estratégia Saúde da Família (ESF) com o desenvolvimento de atividades como: visita domiciliar; grupos de educação em saúde; reunião de equipe com agentes comunitários; planejamento local com sala de situação; e territorialização;

- trabalho em equipe multiprofissional e de caráter interdisciplinar que envolva profissionais e estudantes, favorecendo a integração das ações de saúde e atividades didático- pedagógicas no âmbito local;

- educação permanente, envolvendo trabalho coletivo e pesquisa integrada;

- ações socioeducativas mobilizadoras da comunidade para fortalecer os canais de expressão do controle social.

\section{Dinâmica do Funcionamento}

Com esse entendimento, a RDA se propõe a subsidiar a formação e capacitação de profissionais no âmbito da saúde a partir de programas articulados entre a UFSC e a SMS de Florianópolis, sob uma perspectiva humanista, de excelência técnica e em 
um contexto interdisciplinar, impulsionados pelas ações indutoras do Ministério da Saúde/Ministério de Educação, mediante os projetos Pró-Saúde I e Pró-Saúde II, principalmente.

Sua operacionalização está sedimentada em um regimento próprio, construído coletivamente pelos sujeitos envolvidos em várias oficinas realizadas em 2006 e 2007.

A RDA de Florianópolis integra gestores do ensino e da assistência, estudantes, professores da UFSC e profissionais da SMS de Florianópolis, em um processo de ensino-aprendizagem no qual todas as unidades são cenários potenciais de ensino e de prestação de serviços de saúde, com representação do Conselho Municipal de Saúde (CMS).

\section{Estratégias para operacionalização}

A gestão está composta estruturalmente por um Colegiado Técnico formado por representantes das instituições (cursos da área da saúde incluindo Residência em Medicina de Família e Comunidade e Residência Multidisciplinar em Saúde da Família), Distritos Sanitários de Saúde do Município, supervisores locais, direção CCS, Departamento de Integração Ensino Serviço da SMS de Florianópolis, estudantes e membros do CMS que integram esta Rede Docente Assistencial (RDA), e de um Colegiado de Gestores, constituído pelo secretário municipal de Saúde de Florianópolis, diretor do CCS da UFSC, diretor do HU/UFSC e o coordenador do Colegiado Técnico da RDA.

Além disso, fazem parte do Colegiado Técnico as comissões didático-pedagógica-assistenciais, a comissão de pesquisa e extensão, a comissão de gestão de recursos e uma comissão de educação permanente, escolhida entre seus membros com finalidade de organizar, apoiar e fomentar as atividades que lhes competem.

Atualmente, estão envolvidos na RDA quatro Unidades de Ensino da UFSC: o CCS, com os cursos de graduação em Medicina, Enfermagem, Nutrição, Farmácia/Análises Clínicas, Odontologia, Fonoaudiologia; o Programa da Residência em Medicina de Família e Comunidade e Residência Multidisciplinar em Saúde da Família; Centro de Desportos - curso de Educação Física; Centro de Filosofia e Humanas - curso de Psicologia; Centro Sócio-Econômico - curso de Serviço Social.

Realizam-se reuniões com periodicidade mínima mensal, envolvendo todos os atores para a tomada de decisões coletivas, e planeja-se a realização de seminários semestrais, para avaliação do processo de integração ensino-serviço.

\section{RESULTADOS E IMPACTO OBTIDO}

Diante do exposto, embora ainda precocemente, pode-se afirmar que o processo de construção coletiva sobre os pressupostos de trabalhar em rede e as estratégias estabelecidas para equacionar os problemas identificados, como um compromisso coletivo, fortaleceram a parceria que já se traduz nos seguintes aspectos:

- Compromisso interinstitucional encaminhando politicamente a proposta de criação da RDA, oficializando-a e apoiando a sua institucionalização por meio de um convênio formalizado em 2006.

- Desenvolvimento de gestão compartilhada, estabelecendo atribuições e compromissos das instituições envolvidas a partir de um regimento específico.

- Construção coletiva do processo, respeitando as especificidades históricas e políticas de cada instituição.

- Criação de espaços necessários para discussão e encaminhamento de questões e conflitos inerentes ao processo de consolidação da RDA - oficinas de educação permanente.

- Promoção de um processo de ensino contextualizado na realidade da prática.

- Desenvolvimento de pesquisas integradas, considerando o contexto sociocultural, compatibilizando o modelo pedagógico-assistencial e as necessidades de saúde da população.

Como ilustração, são apresentados os gráficos 1 e 2, que demonstram a evolução da inserção dos alunos dos diferentes cursos na rede municipal de saúde, após a implantação dos projetos Pró-Saúde e PET-Saúde. Pode-se informar que, no ano de 2005, 18 CSs eram campos de prática para o processo de ensino de 647 estudantes e, em 2010, trabalhou-se nessa parceria em 37 CSs e em seis outras Unidades de Saúde, entre Centros de Atenção Psicossocial (Caps), Unidades de Pronto-Atendimento (UPAs) e Policlínicas Municipais, com a participação de 1.422 estudantes.

As instituições de ensino buscam ampliar e aprofundar o conhecimento científico, tecnológico, filosófico e artístico por meio da produção e socialização de pesquisas. Nessa perspectiva, ressalta-se o importante papel da UFSC, a partir de seus cursos de graduação e pós-graduação, na realização de pesquisas necessárias ao desenvolvimento efetivo e equitativo do Sistema de Saúde, considerando as prioridades e realidades epidemiológicas e sanitárias de forma que aumente a possibilidade de que o conhecimento gerado seja efetivamente incorporado a políticas e ações de saúde pública.

Destaca-se que, no período de 2006 a 2009, foram realizados, no âmbito da SMS de Florianópolis, 189 projetos de pesquisa oriundos da UFSC, dentre eles: teses, dissertações, trabalhos de conclusão de curso, projetos com financiamento CNPq e de extensão, dos quais muitos contaram com a participação dos profissionais da SMS de Florianópolis. 


\section{GrÁFICO 1}

Série histórica de alunos na Rede Municipal de Florianópolis por curso

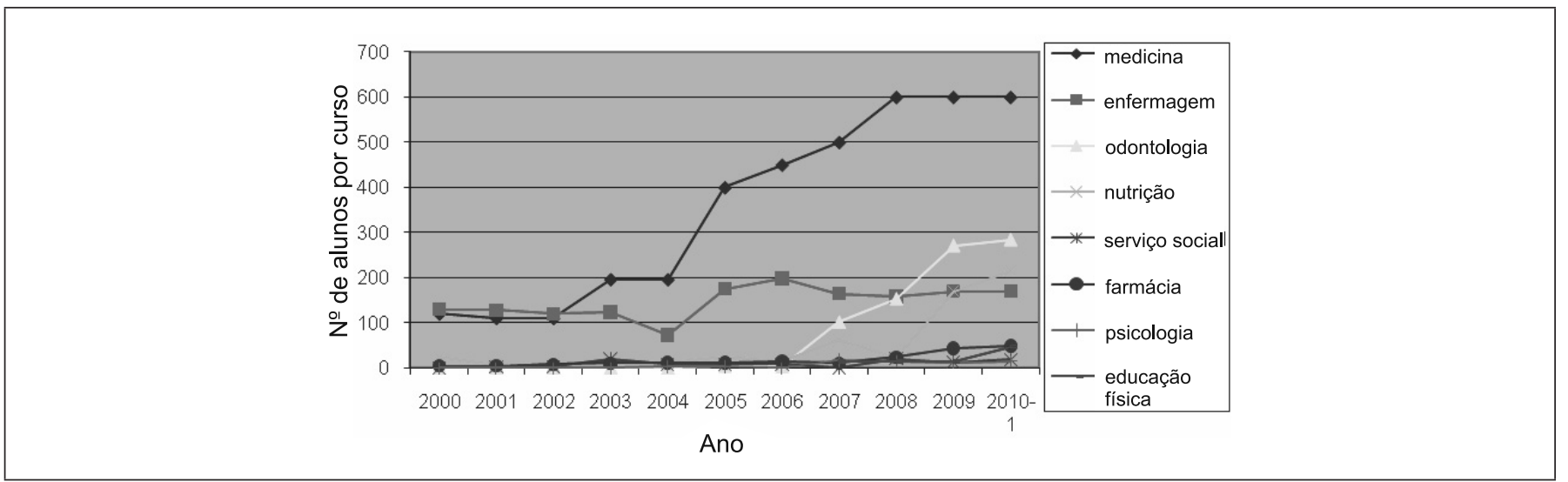

GRÁFICO 2

Série histórica de alunos na Rede Municipal de Florianópolis

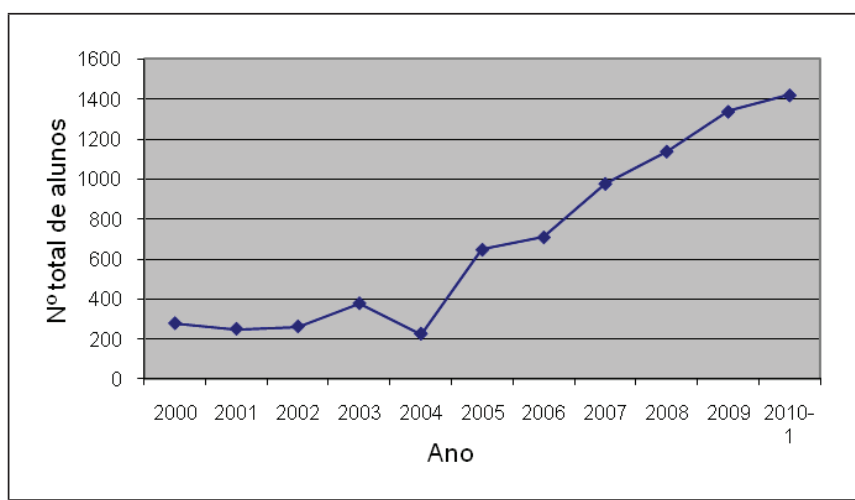

A ampliação do número de alunos nos CSs é compatível com a expansão da área geográfica de atuação, envolvendo mais CSs na perspectiva do alargamento da assistência e na diversidade de experiências durante o processo de formação, conforme é apresentado no quadro 1.

Esses resultados traduzem o impacto das ações que, associadas à implantação dos grupos PET-Saúde da Família, fortalecem os CSs como cenários de prática para os estudantes, possibilitando a integração ensino-serviço e teoria e prática, a partir da realidade apresentada. Isso resulta em processos de trabalho articulados que se diferenciam somente por sua especificidade, aumentando a atuação dos preceptores e docentes. Além disso, reduziu consideravelmente a concentração do número de estudantes nos CSs e compatibilizou o ensino e a pesquisa com a assistência à saúde da população.
QuAdro 1

Cenários de prática 2010 - UFSC/SMS de Florianópolis

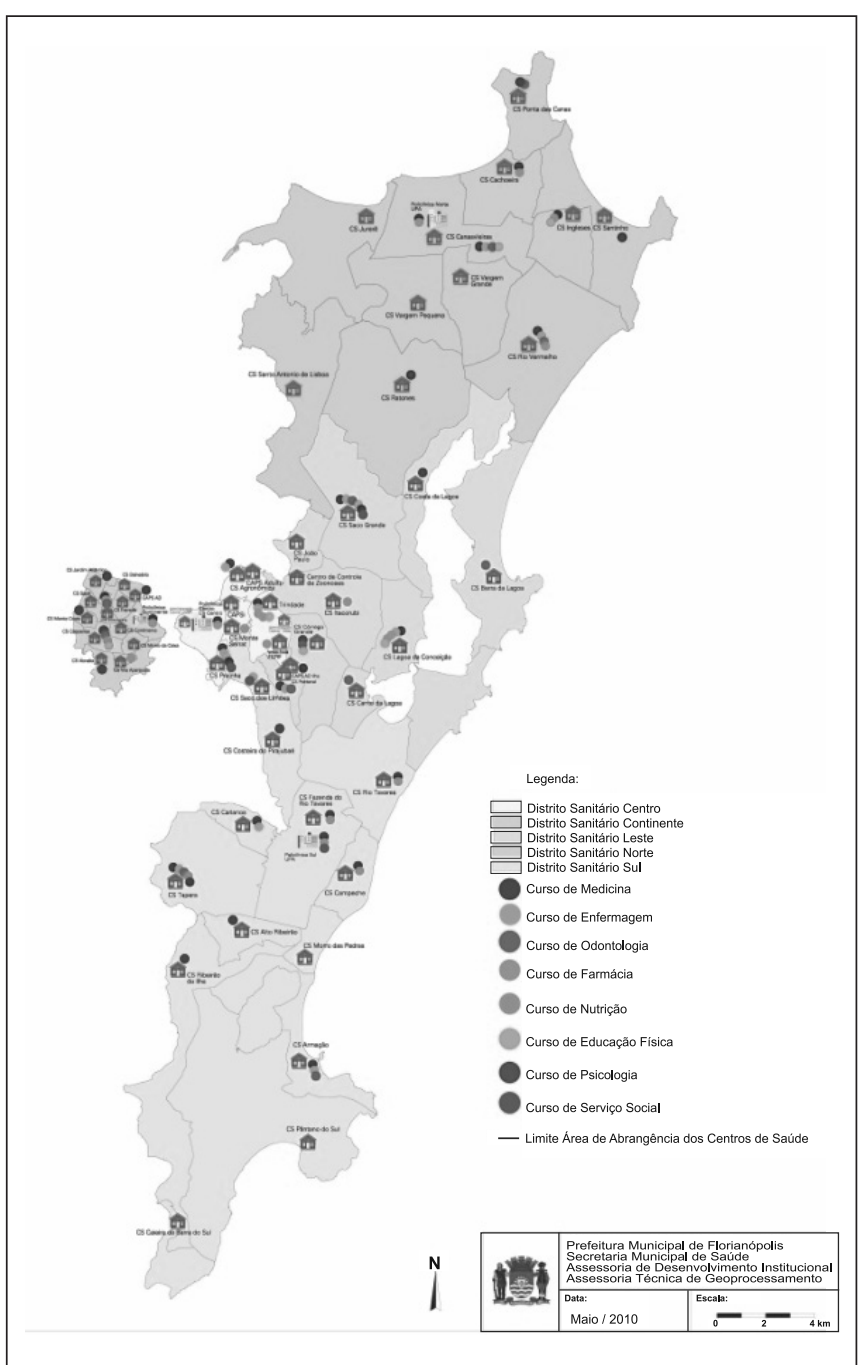




\section{REFLETINDO SOBRE O PROCESSO DE TRABALHAR EM REDE}

A realidade apresenta alguns obstáculos, tais como as estruturas de ensino e de assistência e os contextos de cada curso (alguns em início de discussão e outros já com propostas concretas de integração e articulação). Tal fato determina condições nem sempre favoráveis ao cumprimento das finalidades; contudo, exige paciência, empenho e compromisso para que o trabalho evolua de modo mais integrado. Fortalecendo essa proposta macro de integração coletiva dos cursos da área da saúde da UFSC juntamente com a SMS de Florianópolis, pretende-se assumir o desafio da construção de projetos compartilhados na formação dos profissionais, na perspectiva do fortalecimento do SUS.

Lidar e problematizar o cotidiano do trabalho é um ato complexo e exige revisão constante e interdisciplinar. Dessa maneira, discussões coletivas estão sendo realizadas para a consolidação de um macroprojeto para a formação de um Centro de Referência em Formação para o SUS (título do projeto Pró-Saúde II), cujo objetivo é:

\begin{abstract}
articular as atividades de formação para o SUS, ressaltando as potencialidades de cada área do conhecimento (no ensino, na pesquisa e na extensão), favorecendo a integração entre os cursos, otimizando as ações, ampliando conhecimentos e estimulando o trabalho coletivo e uma maior integração ensino-serviço ${ }^{9}$.(p.3)
\end{abstract}

Esse desafio, pactuado com a criação da RDA, pretende, respeitando as especificidades de cada um, promover ações integradoras entre a Universidade e os serviços que possibilitem ao acadêmico vivenciar na prática a experiência de um trabalho coletivo em saúde, seja nos Centros de Saúde ou nas unidades de internação hospitalar. Além disso, continuar com a reorientação das pesquisas desenvolvidas na área da saúde, mantendo a ênfase na investigação das necessidades da comunidade, organização e financiamento dos serviços de saúde, experimentação de novos modelos de intervenção e avaliação da incorporação de novas tecnologias.

Nesse processo contínuo de discussões associadas aos projetos Pró-Saúde e PET-Saúde, diretrizes gerais estão sendo constantemente pactuadas entre os cursos da UFSC e a SMS de Florianópolis, dentre as quais se destacam:

- Rearticular as matrizes curriculares dos cursos em uma perspectiva usuário-centrada.

- Programar discussões conjuntas sobre a integralidade, envolvendo as diversas áreas (saúde, ciências humanas e sociais, entre outras) na avaliação dos projetos políticos pedagógicos para consolidar o perfil dos profissionais.
- Promover ações socioeducativas mobilizadoras da comunidade para fortalecer os canais de expressão do controle social.

- Conhecer e compreender o papel de cada uma das profissões na equipe de saúde, visando à integralidade do cuidado (reconhecer o núcleo comum e o núcleo específico de cada profissão).

- Desenvolver atividades com enfoque na Estratégia Saúde da Família, tais como: visita domiciliar, grupos de educação em saúde, reunião de equipe com agentes comunitários, planejamento local com sala de situação e territorialização.

- Promover encontros de formação coletiva com ações de Educação Permanente, envolvendo trabalho coletivo e pesquisa integrada; capacitações pedagógicas; seminários; e encontros com os três atores (academia, gestão e movimentos sociais) para formação articulada.

- Criar espaços de encontro e identificação das potencialidades e limitações das diferentes profissões para construção de estratégias interdisciplinares de mudança e inovação nos serviços e no ensino.

- Trabalhar em equipe multiprofissional e de caráter interdisciplinar, que envolva profissionais e estudantes, favorecendo a integração das ações de saúde e atividades didático-pedagógicas que ocorrem no âmbito local, proporcionando vivências coletivas dos acadêmicos dos diferentes cursos, por exemplo, em planejamento comunitário, disciplinas comuns como territorialização e atividades práticas.

Com os programas indutores da reorientação profissional do Ministério da Saúde e Ministério da Educação (Promed, Pró-Saúde e PET-Saúde), fortaleceu-se a função social da educação superior e consolidou-se as parcerias interinstitucionais já existentes em Florianópolis desde a década de 70.

Esses programas, de extrema importância para o direcionamento da formação e da assistência, promoveram o trabalho em equipe multiprofissional; qualificaram a assistência à saúde e o ensino na Atenção Primária à Saúde; e contribuíram para a resolução de problemas prioritários e melhoria dos indicadores de saúde. Com o incentivo e a aproximação cada vez mais efetiva promovida pelos programas ministeriais, os avanços vão se concretizando ao longo do tempo.

Em 2008, é estabelecida, por meio do convênio entre a UFSC e a SMS de Florianópolis, a Farmácia Escola, que dispensa medicamentos da Assistência Farmacêutica na Atenção Básica e do Componente Especializado aos usuários de Florianópolis e realiza atividades de ensino, pesquisa e extensão do curso de Farmácia e Análises Clínicas. 
Em 2009, a SMS de Florianópolis oficializa, via organograma institucional, o Departamento de Integração Ensino Serviço, com o objetivo de: articular e participar das relações interinstitucionais e intrainstitucionais de integração ensino serviço; promover o serviço, a pesquisa e a extensão articulados às necessidades do serviço e comunidade, favorecendo a ampliação da atenção à saúde de qualidade e propiciando a formação dos profissionais da saúde voltada para os princípios do SUS; e colaborar na formação e capacitação de profissionais no âmbito da saúde, através de programas articulados entre a SMS e Instituição de Ensino Superior (IES).

Dessa forma, a RDA se constrói cotidianamente, com negociações e compromissos que se fundamentam em atividades que se articulam para o alcance das metas, respeitando as suas especificidades e fortalecendo as ações coletivas na perspectiva da integração ensino-serviço, para o fortalecimento dos princípios do SUS.

Portanto, a proposta metodológica de trabalhar em rede concretiza os princípios da educação permanente, associando teoria e prática, orientando a seleção de conteúdos curriculares para os problemas sociais e epidemiologicamente relevantes. Dessa forma, consegue-se ampliar a formação para além da informação técnico-científica, ressaltando a dimensão ética e política como eixo essencial para o exercício profissional ${ }^{10}$.

Contudo, essa proposta só se efetiva tendo em vista a vontade política dos gestores nacionais e locais, o compromisso pessoal e institucional com o processo de formação dos profissionais da saúde e a responsabilidade com a melhoria da qualidade da assistência à saúde do município. Segundo Merhy ${ }^{11}$,

"[...] somos em certas situações, a partir de certos recortes, sujeitos de saberes e das ações que nos permitem agir protagonizando processos novos como força de mudança. Mas, ao mesmo tempo, sob outros recortes e sentidos, somos reprodutores de situações dadas [...] mesmo protagonizando certas mudanças, em muito conservamos. Entretanto, somos responsáveis pelo que fazemos [...] precisamos nos reconhecer em nossos afazeres".(p.14)

\section{REFERÊNCIAS}

1. Brasil. Ministério da Saúde. Pró-Saúde: programa nacional de reorientação da formação profissional em saúde / Ministério da Saúde, Ministério da Educação - Brasília: Ministério da Saúde, 2005. 77 p. - (Série C. Projetos, Programas e Relatórios)

2. Assamann H, Sung JM. Competência e sensibilidade solidária: educar para a esperança. Petrópolis: Vozes; 2000.
3. Vazquez AS. Filosofia da práxis. 4 ed. Rio de Janeiro: Paz e Terra; 1986.

4. Franco MLPB. Possibilidades e limites do trabalho enquanto princípio educativo. Cad. Pesq. 1989;(68): 29-37.

5. Reibnitz KS, Prado ML. Processo de trabalho, processo educativo e formação profissional em Enfermagem. In: Reibnitz KS, Prado ML, org. Inovação e Educação em Enfermagem. Florianópolis: Cidade Futura; 2006. p. 79-108.

6. Ceccim RB. Educação permanente em saúde: desafio ambicioso e necessário. Interface Comunic. Saúde, Educ. 2005;9(16): 161-8.

7. Sisson MC. Integração ensino-serviços em Florianópolis. In: Pereima MJL, orgs. Da proposta à ação: currículo integrado do curso de graduação em Medicina da UFSC. Florianópolis: Ed. UFSC; 2005.

8. Souza ML, Reibnitz KS, Cartana MH. Redes de ensino: estratégia de formação para os profissionais de enfermagem. In: Reibnitz KS, org.Educação, trabalho e enfermagem. Módulo III do Curso de Especialização em metodologia do ensino para profissionalização em enfermagem. NFR/ UFSC; 2000.p. 47-65.

9. Universidade Federal de Santa Catarina. Projeto de criação do Centro de Referência em Formação para o SUS. Julho, 2004.

10. Brasil. Ministério da Saúde. Secretaria de Gestão do Trabalho de Educação na Saúde. Departamento de Gestão da Educação na Saúde. A educação permanente entra na roda: polos de educação permanente em saúde: conceitos e caminhos a percorrer. Brasília: MS; 2005.

11. Merhy EE. Saúde: a cartografia do trabalho vivo. São Paulo: Hucitec; 2002.

\section{CONTRIBUIÇÃO DOS AUTORES}

Kenya Schmidt Reibnitz, Maria Francisca dos Santos Daussy, Carlos Alberto Justo da Silva, Marynes Terezinha Reibnitz e Daiana Kloh participaram da redação e revisão de todas as partes do artigo.

\section{CONFLITO DE INTERESSES}

Declarou não haver.

\section{ENDEREÇO PARA CORRESPONDÊNCIA}

Kenya Schmidt Reibnitz

Cenro de Ciencias da Saúde

Campus Universitário - Trindade - Florianópolis

CEP. 88040-900 SC

E-mail: kenya@ccs.ufsc.br 